\title{
BANACH SPACES WITH SEPARABLE DUALS SUPPORT DUAL HYPERCYCLIC OPERATORS
}

\author{
HÉCTOR N. SALAS \\ Department of Mathematics, University of Puerto Rico, Mayagüez, Puerto Rico 00681 \\ e-mail: salas@math.uprm.edu
}

(Received 4 August, 2006; revised 3 January and 13 March, 2007; accepted 19 March, 2007)

\begin{abstract}
Let $E$ be a Banach space such that its dual $E^{*}$ is separable. We show that there exists a hypercyclic bounded operator $T$ on $E$ such that its adjoint $T^{*}$ is also hypercyclic on $E^{*}$. We also exhibit a new kind of dual hypercyclic operator. Thus answers affirmatively two of the questions raised by Henrik Petersson in a recent paper.

2000 Mathematics Subject Classification. Primary 47A16, 47A05, Secondary. $47 \mathrm{~B} 37$.
\end{abstract}

1. Introduction. Pelczyński, in his review of Rolewicz's paper [16] wrote "In a separable Hilbert space $H$ the author constructs a bounded linear operator, say $A$, with the following strange property: there exists an element $x$ in $H$ such that the set $\left\{A^{n} x: n=0,1, \ldots\right\}$ is dense in $H$ ". This "strange" property is what nowadays is called hypercyclicity and can no longer be described with this adjective.

A good place to learn the basic subject matter and its rich roots is the paper by Godefroy and Shapiro [8]. The survey by Grosse-Erdmann [11] is quite comprehensive. It also has an extensive bibliography which he updates regularly on his website.

In a preliminary version of [14], Herrero posed as Problem 2 whether or not there exists an operator $T$ on a Hilbert space such that $T$ and its adjoint $T^{*}$ are both hypercyclic. This was answered affirmatively in [17] and such an operator was a bilateral weighted shift. In [15], Petersson studied this property in the more general context of Banach spaces. Naturally, if the topology of the dual space is given by its norm, one should restrict the attention to Banach spaces with separable duals. Petersson called operators $T$ with this property, that both $T$ and $T^{*}$ are hypercyclic, dual hypercyclic operators. He showed that any Banach space with a shrinking symmetric basis supports such an operator; which is, essentially, a bilateral weighted shift with respect to such a basis. He observed that one also obtains such operators on spaces with a symmetric basis if one considers the dual space endowed with the weak* topology.

In Section 2 we obtain some preliminary results. In Section 3 we answer Question 2 of [15]: Does every infinite dimensional separable Banach space with separable dual support a dual hypercyclic operator? In Section 4 we answer Question 1 of [15]: Does there exist a dual hypercyclic operator that is not of the form of Theorem 1? Finally, in Section 5, we indicate some questions and problems.

2. Preparation. Let $E$ be a separable complex Banach space and let $\mathcal{B}(E)$ be the set of bounded linear operators on $E$. Let $\left\{x_{n}: n \in \mathbb{Z}\right\}$ be a subset of $E$. For a subset $A$ of the integers, the set $\left[x_{j}: j \in A\right]$ denotes the closed subspace spanned by $\left\{x_{j}: j \in A\right\}$. 
The system $\left\{\left(x_{n}, x_{n}^{*}\right): x_{n} \in E, x_{n}^{*} \in E^{*}, n \in \mathbb{Z}\right\}$ is called biorthogonal if $x_{n}^{*}\left(x_{m}\right)=$ $\delta_{n}^{m}$. If, in addition, $\left[x_{n}: n \in \mathbb{Z}\right]=E$ and $E^{*}$ is the weak* closure of the linear span of $\left\{x_{n}^{*}\right.$ : $n \in \mathbb{Z}\}$, then $\left\{x_{n}: n \in \mathbb{Z}\right\}$ is called a Markushevich basis. Note that the (biorthogonal) functionals $\left\{x_{n}^{*}: n \in \mathbb{Z}\right\}$ are unique for such a basis $\left\{x_{n}: n \in \mathbb{Z}\right\}$.

Ovsepian and Pelczyński (see p. 44 of [13]) showed that every separable Banach space has a Markushevich basis in which

$$
\left\|x_{n}\right\|=1 \text { for all } n \text { and } \sup _{n}\left\|x_{n}^{*}\right\|<\infty \text {. }
$$
$E^{*}$.

Moreover, when $E^{*}$ is separable, $\left\{x_{n}: n \in \mathbb{Z}\right\}$ may be chosen so that $\left[x_{n}^{*}: n \in \mathbb{Z}\right]=$

When $E^{*}$ is separable, we will always consider that property (OP) includes $\left[x_{n}^{*}: n \in\right.$ $\mathbb{Z}]=E^{*}$.

Recall that for $y \in E$ and $y^{*} \in E^{*}$, the tensor product $y^{*} \otimes y \in \mathcal{B}(E)$ is defined by $y^{*} \otimes y(x)=y^{*}(x) y$, and $\left\|y^{*} \otimes y\right\| \leq\left\|y^{*}\right\|\|y\|$. Also $\left(y^{*} \otimes y\right)^{*}=y \otimes y^{*}$ if we identify $y$ with $i(y)$ where $i: E \longrightarrow E^{* *}$ is the canonical injection.

Proposition 2.1. Let $E$ be a Banach space with Markushevich basis $\left\{x_{n}: n \in \mathbb{Z}\right\}$ which satisfies (OP). Let $w_{n}>0$ for all $n$ and $\sum_{n \in \mathbb{Z}} w_{n}<\infty$. Then the "bilateral weighted shift" $T=\sum_{n \in \mathbb{Z}} w_{n} x_{n}^{*} \otimes x_{n-1}$ is compact and quasinilpotent. Its adjoint $T^{*}$ is $\sum_{n \in \mathbb{Z}} w_{n} x_{n-1} \otimes x_{n}^{*}$.

Proof. $T$ is the norm limit of finite rank operators and therefore compact. Thus if $\lambda \neq 0$ is in the spectrum of $T, \sigma(T)$, then $\lambda$ is an eigenvalue. Let $x$ be a corresponding eigenvector. Then $x \notin\left[x_{j}: j \in A\right]$ whenever $A$ is a finite set.

Since $\lambda x=\sum_{n \in \mathbb{Z}} w_{n} x_{n}^{*}(x) x_{n-1}$ it follows that $\lambda x_{n-1}^{*}(x)=w_{n} x_{n}^{*}(x)$. But $x$ is not zero and there is $n$, say $n=0$, for which $x_{n}^{*}(x) \neq 0$. Therefore

$$
x_{n}^{*}(x)=\frac{\lambda^{n}}{w_{1} \ldots w_{n}} x_{0}^{*}(x) \neq 0
$$

for all $n>0$. This is impossible since $\sup _{n}\left\|x_{n}^{*}\right\|=M<\infty$ but

$$
\left\|x_{n}^{*}\right\| \geq \frac{|\lambda|^{n}}{w_{1} \ldots w_{n}} \frac{\left|x_{0}^{*}(x)\right|}{\|x\|} \rightarrow \infty
$$

because $\sum w_{n}<\infty$.

The last part follows from

$$
\left(\sum_{|n| \leq N} w_{n} x_{n}^{*} \otimes x_{n-1}\right)^{*}=\sum_{|n| \leq N} w_{n} x_{n-1} \otimes x_{n}^{*}
$$

and taking limit for $N \rightarrow \infty$.

REMARK 2.2. Let $T$ be a bilateral weighted shift with respect to an orthonormal basis of a Hilbert space. The spectrum of $T$ is always centered at the origin. There are these three possibilities (i) $\sigma(T)=\{0\}$, (ii) $\sigma(T)$ is a disc, or (iii) $\sigma(T)$ is an annulus. This is due to Kelley [19, p116]. These possibilities are also the only ones in the case that $T$ is a bilateral weighted shift with respect to the canonical basis of $\ell^{p}(\mathbb{Z})$, with $1 \leq p<\infty$, or $c_{0}(\mathbb{Z})$. 
Recall that a Schauder basis $\left\{e_{n}: n \in \mathbb{Z}\right\}$ of a Banach space $E$ is unconditional if and only if $\left\{e_{\pi(n)}: n \in \mathbb{Z}\right\}$ also forms a basis for any permutation $\pi$ of $\mathbb{Z}$. It is a symmetric basis if, in addition, all $\left\{e_{\pi(n)}: n \in \mathbb{Z}\right\}$, where $\pi$ is a permutation, are equivalent, p113 of [13].

Proposition 2.3. Let $\left\{e_{n}: n \in \mathbb{Z}\right\}$ be a symmetric basis of $E$ with corresponding biorthogonal functionals $\left\{e_{n}^{*}: n \in \mathbb{Z}\right\}$ and let $\left\{w_{n}: n \in \mathbb{Z}\right\}$ be a positive bounded sequence. If $T=\sum_{n \in \mathbb{Z}} w_{n} e_{n}^{*} \otimes e_{n-1}$, then $\sigma(T)$ has circular symmetry.

Proof. $T$ is bounded by Lemma 1 of [15]. Since $\sigma(T)$ consists of the union of the approximate spectrum and the compression spectrum, it is enough to see that each of these sets has circular symmetry. Let $\lambda$ be an approximate eigenvalue of $T$ and let $\left\|(T-\lambda I) \sum_{n \in \mathbb{Z}} a_{n} e_{n}\right\|<\epsilon$ with $\left\|\sum_{n \in \mathbb{Z}} a_{n} e_{n}\right\|=1$.

Let $K$ be the symmetric basis constant (which is greater than or equal to the unconditional basis constant) and $|z|=1$. Then, by Proposition 1.c.7 of [13],

$$
(2 K)^{-1} \leq\left\|\sum_{n \in \mathbb{Z}} z^{n} a_{n} e_{n}\right\| \leq 2 K
$$

and

$$
\left\|(T-z \lambda I) \sum_{n \in \mathbb{Z}} z^{n} a_{n} e_{n}\right\|<2 K \epsilon .
$$

Since $0<\epsilon$ is arbitrary, $z \lambda \in \sigma(T)$.

We now show that if the range of $T-\lambda I$ is dense in $E$, so is the range of $T-z \lambda I$ for $|z|=1$. It is enough to show that if $z^{-k-1} e_{k}$ is in the closure of the range of $T-\lambda I$, then $e_{k}$ is in the closure of the range of $T-z \lambda I$. But if

$$
\left\|(T-\lambda I)\left(\sum_{n \in \mathbb{Z}} a_{n} e_{n}\right)-z^{-k-1} e_{k}\right\|<\epsilon,
$$

then

$$
\left\|(T-z \lambda I)\left(\sum_{n \in \mathbb{Z}} z^{n} a_{n} e_{n}\right)-e_{k}\right\|<2 K \epsilon .
$$

We still need a few more observations.

Proposition 2.4. Let $F$ be a finite dimensional Banach space with basis $\left\{e_{j}: 0 \leq\right.$ $\left.j \leq 2^{k+1}-1\right\}$. Define $L: F \longrightarrow F$ by $L\left(e_{0}\right)=0$ and $L\left(e_{j}\right)=w_{j} e_{j-1}$ for $0<j$ and $w_{j}>0$. Let $x_{1}, \ldots, x_{k-1}$ and $z_{k}$ be in $\left[e_{0}, \ldots, e_{2^{k}-1}\right]$. Then for each pair $\delta>0$ and $\epsilon>0$ there exist $x_{k}$ in $\left[e_{2^{k}}, \ldots, e_{2^{k+1}-1}\right]$ and $n \in \mathbb{N}$ such that

$$
\left\|x_{k}\right\|<\delta \text { and }\left\|(I+L)^{n}\left(\sum_{j=1}^{k} x_{j}\right)-z_{k}\right\|<\epsilon .
$$

Proof. When $F$ is a Hilbert space this is the key step in the proof of Theorem 3.3 of [18]. Since all norms are equivalent on a finite dimensional space the result follows at once. 
COROLlaRY 2.5. Let $T \in \ell^{2}(\mathbb{N})$ be a unilateral backward weighted shift with positive weights. Then the $k$ direct sum $(I+T) \oplus \ldots \oplus(I+T)$ is hypercyclic.

Proof. We sketch the proof when $k=2$.

In Proposition 2.4 for $x_{1}, \ldots, x_{k-1}, z_{k}$ and $y_{1}, \ldots, y_{k-1}, \hat{z}_{k}$, we can, again by looking at the proof of the above mentioned Theorem 3.3, obtain $x_{k}$ and $y_{k}$ and the same $n$ such that (1) holds for $L=T$ and

$$
\left\|y_{k}\right\|<\delta \text { and }\left\|(I+T)^{n}\left(\sum_{j=1}^{k} y_{j}\right)-\hat{z}_{k}\right\|<\epsilon .
$$

If $\left\{z_{k} \oplus \hat{z}_{k}: k \in \mathbb{N}\right\}$ is dense in $\ell^{2}(\mathbb{N}) \oplus \ell^{2}(\mathbb{N})$ and the $x_{j}$ 's are chosen with disjoint support (and the same for the $y_{j}$ 's), then we proceed as in Theorem 3.3 of [18] to obtain a hypercyclic vector of the form

$$
\sum_{i=1}^{\infty} x_{i} \oplus \sum_{i=1}^{\infty} y_{i}
$$

COROLlary 2.6. Let $T \in \ell^{2}(\mathbb{N})$ be a unilateral backward weighted shift with positive weights. Then I $+T$ satisfies the Hypercyclicity Criterion.

Proof. Bès and Peris showed the equivalence of the Hypercyclicity Criterion with the hypercyclicity of the direct sums, see Theorem 2.3 of [6].

REMARK 2.7. In Section 4 of [12], León-Saavedra and Montes-Rodríguez proved directly that $I+T$ satisfies the Hypercyclicity Criterion. Moreover, Grivaux in Lemma 2.3 of $[\mathbf{1 0}]$ proved that $I+T$ is mixing.

The following lemma will be used in the inductive step of the next theorem. Observe that any backward bilateral weighted shift can be seen as a forward bilateral weighted shift by relabelling the vectors in the basis (via $n \rightarrow-n$ for $n \in \mathbb{Z}$ ). Thus Lemma 2.8 can also be applied to forward bilateral shifts.

LEMMA 2.8. Suppose that $E$ is a Banach space with Markushevich basis $\left\{e_{n}: n \in \mathbb{Z}\right\}$ which satisfies (OP). Let $w_{n}>0$ for all $n$ and $\sum_{n \in \mathbb{Z}} w_{n}<\infty$. For s fixed, let $x_{1}, \ldots, x_{k-1}$ and $z_{k}$ be in $\left[e_{s}, \ldots, e_{s+2^{k}-1}\right]$. Then for each pair $\delta>0$ and $\epsilon>0$ there exist $x_{k}$ in $\left[e_{s+2^{k}}, \ldots, e_{s+2^{k+1}-1}\right]$ and $n \in \mathbb{N}$ and $0<v_{s} \leq w_{s}$ such that $x_{k}$ and the bilateral weighted shift

$$
T=\sum_{n \in \mathbb{Z} \backslash\{s\}} w_{n} e_{n}^{*} \otimes e_{n-1}+v_{s} e_{s}^{*} \otimes e_{s-1}
$$

satisfy

$$
\left\|x_{k}\right\|<\delta \text { and }\left\|(I+T)^{n}\left(\sum_{i=1}^{k} x_{i}\right)-z_{k}\right\|<\epsilon .
$$

Proof. Set for a moment $v_{s} \leq w_{s}$ and consider the operator $T-v_{s} e_{s}^{*} \otimes e_{s-1}$. Call $L$ the restriction of this operator to the invariant subspace $\left[e_{s}, \ldots, e_{s+2^{k+1}-1}\right]$. By 
Proposition 2.4 we have $x_{k}$ in $\left[e_{s+2^{k}}, \ldots, e_{s+2^{k+1}-1}\right]$ and $n \in \mathbb{N}$ such that

$$
\left\|x_{k}\right\|<\delta \text { and }\left\|(I+L)^{n}\left(\sum_{i=1}^{k} x_{i}\right)-z_{k}\right\|<\frac{\epsilon}{2} .
$$

Let $P$ be the projection whose kernel is $\left[e_{s}, \ldots, e_{s+2^{k+1}-1}\right]$ and range is $\left[e_{s-n}, \ldots, e_{s-1}\right]$. Then $P\left((I+T)^{n}\left(\sum_{i=1}^{k} x_{i}\right)-z_{k}\right)$ is

$$
\sum_{j=s}^{s+2^{k+1}-1} \sum_{m=j-s+1}^{n}\left(\begin{array}{l}
n \\
m
\end{array}\right) T^{m}\left(\sum_{i=1}^{k} e_{j}^{*}\left(x_{i}\right) e_{j}\right) .
$$

$\left(P \in \mathcal{B}\left(\left[e_{s-n}, \ldots, e_{s+2^{k+1}-1}\right]\right)\right.$ where dimension of $\left[e_{s-n}, \ldots, e_{s+2^{k+1}-1}\right]$ is $n+2^{k+1}$.)

Set $Q=1+\sup _{n} w_{n}$. Then for $j-s<m$ we have $\left\|T^{m}\left(e_{j}\right)\right\|<v_{s} Q^{m-1}$. Set also

$$
M=\max \left\{\left|e_{j}^{*}\left(x_{i}\right)\right|: s \leq j<s+2^{k+1} ; 1 \leq i \leq k\right\} .
$$

Finally, let $v_{s}$ be such that $v_{s} k M 2^{k+1}(1+Q)^{n}<\frac{\epsilon}{2}$.

Then

$$
\begin{aligned}
& \left\|\sum_{j=s}^{s+2^{k+1}-1} \sum_{m=j-s+1}^{n}\left(\begin{array}{c}
n \\
m
\end{array}\right) T^{m}\left(\sum_{i=1}^{k} e_{j}^{*}\left(x_{i}\right) e_{j}\right)\right\| \\
& \leq v_{s} k M 2^{k+1} \sum_{m=1}^{n}\left(\begin{array}{c}
n \\
m
\end{array}\right) Q^{m} \leq v_{s} k M 2^{k+1}(1+Q)^{n}<\frac{\epsilon}{2} .
\end{aligned}
$$

Therefore (2) and (3) imply that

$$
\begin{gathered}
\left\|(I+T)^{n}\left(\sum_{j=1}^{k} x_{j}\right)-z_{k}\right\|<\left\|(I+L)^{n}\left(\sum_{j=1}^{k} x_{j}\right)-z_{k}\right\| \\
+\left\|\sum_{j=s}^{s+2^{k+1}-1} \sum_{m=j-s+1}^{n}\left(\begin{array}{l}
n \\
m
\end{array}\right) T^{m}\left(\sum_{i=1}^{k} e_{j}^{*}\left(x_{i}\right) e_{j}\right)\right\|<\epsilon .
\end{gathered}
$$

3. Existence of dual hypercyclic operators. We now have all the ingredients for proving the main result. This answers affirmatively Question 2 of [15]. The idea of the proof is to start out with an operator like the one given in Proposition 2.1, say $L_{0}$, and then modify the weights, one at a time, in the order suggested in the statement of the theorem. Call the operator so obtained $T$. Our candidate for a dual hypercyclic operator is $I+T$.

THEOREM 3.1. Suppose that $E$ is a Banach space whose dual is separable and with Markushevich basis $\left\{e_{n}: n \in \mathbb{Z}\right\}$ which satisfies (OP). Let $w_{n}>0$ for all $n$ and $\sum_{n \in \mathbb{Z}} w_{n}<\infty$. Then there exists a dual hypercyclic operator

$$
I+\sum_{n \in \mathbb{Z}} v_{n} e_{n}^{*} \otimes e_{n-1}=I+T
$$


such that all $v_{n}>0$ and $v_{n}=w_{n}$ except, possibly, for $v_{0} \leq w_{0}, \quad v_{4} \leq w_{4}, \quad v_{-4} \leq$ $w_{-4}, v_{12} \leq w_{12}$ and for $k>2$

$$
v_{-\left(4+\sum_{i=2}^{k-1} 2^{2 i}\right)} \leq w_{-\left(4+\sum_{i=2}^{k-1} 2^{2 i}\right)} \quad \text { and } \quad v_{4+\sum_{i=2}^{k} 2^{2 i-1}} \leq w_{4+\sum_{i=2}^{k} 2^{2 i-1}} .
$$

Proof. Since (OP) is in force, we have that $\left\|e_{n}\right\|=1$ for $n \in \mathbb{Z}$ and $\sup _{n}\left\|e_{n}^{*}\right\|<\infty$ and also $\left[e_{n}^{*}: n \in \mathbb{Z}\right]=E^{*}$. Let $L_{0}=\sum_{n \in \mathbb{Z}} w_{n} e_{n}^{*} \otimes e_{n-1}$, then

$$
\max \left\{\left\|L_{0}\right\|,\left\|L_{0}^{*}\right\|\right\} \leq \sup _{n}\left\|e_{n}^{*}\right\|\left(\sum_{n \in \mathbb{Z}} w_{n}\right)=M .
$$

Let $\left\{z_{k}: k \in \mathbb{N}\right\}$ be dense in $E$ such that $z_{1} \in\left[e_{0}, e_{1}\right]$ and $z_{2} \in\left[e_{j}:-4 \leq j \leq 3\right]$ and for $2<k$

$$
z_{k} \in\left[e_{j}:-\left(4+\sum_{i=2}^{k-1} 2^{2 i}\right) \leq j \leq 4+\sum_{i=2}^{k-1} 2^{2 i-1}-1\right]
$$

In an analogous fashion we choose $\left\{z_{k}^{*}: k \in \mathbb{N}\right\}$ to be dense in $E^{*}$ with $z_{1}^{*} \in\left[e_{0}^{*}, e_{1}^{*}\right]$ and so forth.

Notice that $z_{k}$ is a linear combination of at most $2^{2 k-1}$ consecutive vectors of $\left\{e_{n}: n \in \mathbb{Z}\right\}$. (Similarly for $z_{k}^{*}$.)

In the sequel we will denote by $u_{i}$ the weights of $L_{j}$ and by $v_{i}$ the weights of the new operator $L_{j+1}$.

By Lemma 2.8 there exist $x_{1} \in\left[e_{2}, e_{3}\right]$ and $n_{1} \in \mathbb{N}$ and bilateral backward shift $L_{1}$ such that

$$
\left\|x_{1}\right\|<2^{-1} \text { and }\left\|\left(I+L_{1}\right)^{n_{1}}\left(x_{1}\right)-z_{1}\right\|<2^{-1}
$$

where the weights are the same for operators $L_{0}$ and $L_{1}$ except, possibly, $v_{0} \leq u_{0}=w_{0}$.

For $L_{1}^{*}$, which is a bilateral forward shift on $E^{*}$, there exist, by applying Lemma 2.8 again, $y_{1}^{*} \in\left[e_{-4}^{*}, e_{-3}^{*}, e_{-2}^{*}, e_{-1}^{*}\right]$ and $n_{2}$ and $L_{2}^{*}$ such that

$$
\left\|y_{1}^{*}\right\|<2^{-1} \text { and }\left\|\left(I+L_{2}^{*}\right)^{n_{2}}\left(y_{1}^{*}\right)-z_{1}^{*}\right\|<2^{-1}
$$

where the weights of $L_{1}$ and $L_{2}$ are the same except, possibly, that $v_{4} \leq u_{4}=w_{4}$.

Observe that we also have

$$
\left\|\left(I+L_{2}\right)^{n_{1}}\left(x_{1}\right)-z_{1}\right\|<2^{-1} .
$$

Before going to the inductive step, let us see how $x_{2}$ and $y_{2}$ are chosen.

We choose $x_{2} \in\left[e_{i}: 4 \leq i \leq 11\right]$ and $n_{3} \in \mathbb{N}$ and $L_{3}$ such that

$$
\left\|x_{2}\right\|<2^{-2}(1+M)^{-n_{1}-n_{2}}
$$

and

$$
\left\|\left(I+L_{3}\right)^{n_{3}}\left(x_{1}+x_{2}\right)-z_{2}\right\|<2^{-2},
$$

where the weights of $L_{2}$ and $L_{3}$ are the same except possibly that $v_{-4} \leq u_{-4}=w_{-4}$ and $M$ is as in (4). 
We choose $y_{2}^{*} \in\left[e_{i}^{*}:-20 \leq i \leq-5\right]$ and $n_{4} \in \mathbb{N}$ and $L_{4}$ such that

$$
\left\|y_{2}^{*}\right\|<2^{-2}(1+M)^{-n_{1}-n_{2}-n_{3}}
$$

and

$$
\left\|\left(I+L_{4}^{*}\right)^{n_{4}}\left(y_{1}^{*}+y_{2}^{*}\right)-z_{2}^{*}\right\|<2^{-2},
$$

where the weights of $L_{3}$ and $L_{4}$ are the same except possibly that $v_{12} \leq u_{12}=w_{12}$.

Assume that we have already chosen $x_{1}, \ldots, x_{k-1}$ and $y_{1}^{*}, \ldots, y_{k-1}^{*}$ with norms adequately small and $n_{1}, \ldots, n_{2 k-2} \in \mathbb{N}$ and also $L_{1}, \ldots, L_{2 k-2}$.

By applying Lemma 2.8 once again we can find $x_{k}$ as a linear combination of at most $2^{2 k-1}$ consecutive vectors of $\left\{e_{n}: n \in \mathbb{Z}\right\}$. More precisely

$$
x_{k} \in\left[e_{l}: 4+\sum_{i=2}^{k-1} 2^{2 i-1} \leq l \leq 4+\sum_{i=2}^{k} 2^{2 i-1}-1\right]
$$

and $n_{2 k-1}$ and $L_{2 k-1}$ such that

$$
\left\|x_{k}\right\|<2^{-k}(1+M)^{-\sum_{j=1}^{2 k-2} n_{j}}
$$

and

$$
\left\|\left(I+L_{2 k-1}\right)^{n_{2 k-1}}\left(\sum_{i=1}^{k} x_{i}\right)-z_{k}\right\|<2^{-k}
$$

where $L_{2 k-2}$ and $L_{2 k-1}$ might differ only in that

$$
v_{-\left(4+\sum_{i=2}^{k-1} 2^{2 i}\right)} \leq u_{-\left(4+\sum_{i=2}^{k-1} 2^{2 i}\right)}=w_{-\left(4+\sum_{i=2}^{k-1} 2^{2 i}\right)} .
$$

We then find that

$$
y_{k}^{*} \in\left[e_{l}^{*}:-\left(4+\sum_{i=2}^{k} 2^{2 i}\right) \leq l \leq-\left(4+\sum_{i=2}^{k-1} 2^{2 i}+1\right)\right]
$$

and $n_{2 k}$ and $L_{2 k}$ such that

$$
\left\|y_{k}^{*}\right\|<2^{-k}(1+M)^{-\sum_{j=1}^{2 k-1} n_{j}}
$$

and

$$
\left\|\left(I+L_{2 k}^{*}\right)^{n_{2 k}}\left(\sum_{i=1}^{k} y_{i}^{*}\right)-z_{k}^{*}\right\|<2^{-k},
$$

where $L_{2 k-1}$ and $L_{2 k}$ might differ only in that

$$
v_{4+\sum_{i=2}^{k} 2^{2 i-1}} \leq u_{4+\sum_{i=2}^{k} 2^{2 i-1}}=w_{4+\sum_{i=2}^{k} 2^{2 i-1}} .
$$

Let $T$ be the bilateral backward shift that is the limit in the norm operator topology of $L_{j}$ when $j$ goes to infinity. 
To finish the proof we have to check that $x=\sum_{i=1}^{\infty} x_{i}$ is hypercyclic for $I+T$ while $y^{*}=\sum_{i=1}^{\infty} y_{i}^{*}$ is hypercyclic for $I+T^{*}$.

We prove it just for $x$. We have that

$$
\begin{aligned}
& \left\|(I+T)^{n_{2 k-1}}(x)-z_{k}\right\| \\
& \leq\left\|(I+T)^{n_{2 k-1}}\left(\sum_{i=1}^{k} x_{i}\right)-z_{k}\right\|+\left\|(I+T)^{n_{2 k-1}}\left(\sum_{i=k+1}^{\infty} x_{i}\right)\right\| \\
& \leq 2^{-k}+\sum_{i=k+1}^{\infty}\left\|(I+T)^{n_{2 k-1}}\left(x_{i}\right)\right\| \\
& \quad \leq 2^{-k}+\sum_{i=k+1}^{\infty}(1+M)^{n_{2 k-1}}\left(\left\|x_{i}\right\|\right) \leq \sum_{i=k}^{\infty} 2^{-i} .
\end{aligned}
$$

Thus $(I+T)^{n_{2 k-1}}(x)-z_{k} \rightarrow 0$ as $k \rightarrow \infty$; since $\left\{z_{k}: k \in \mathbb{N}\right\}$ is dense, this implies that $x$ is hypercyclic for $I+T$. The second inequality is because of (6), whereas the last inequality is because of (5).

Recall that a hypercyclic subspace for an operator $T$ is an infinite dimensional closed subspace whose nonzero vectors are hypercyclic.

REMARK 3.2. Corollaries 2.5 and 2.6 are also true for the bilateral shifts constructed in Theorem 3.1, and therefore $I+T$ satisfies the Hypercyclicity Criterion. Since $\sigma(I+$ $T)=\{1\}$, the essential spectrum of $I+T$ meets the unit disk. Then a theorem of González, León-Saavedra and Montes-Rodríguez asserts that $I+T$ has a hypercyclic subspace [9, p178].

4. New dual hypercyclic operators on Hilbert spaces. A similar argument to the one given in the proof of Theorem 3.1 also proves the following theorem, which is also valid for $\ell^{p}(\mathbb{Z})$ and $c_{0}(\mathbb{Z})$ where $1<p<\infty$. The shifts below are considered with respect to the canonical basis. Since $L$ may not be compact, $V$ is the limit in the strong operator topology of the intermediate operators $L_{j}$.

THEOREM 4.1. Let L be a bilateral backward weighted shift on $\ell^{2}(\mathbb{Z})$, with positive bounded weight sequence $\left\{w_{n}\right\}$. Then there exists another bilateral backward shift $V$ on $\ell^{2}(\mathbb{Z})$, with positive weight sequence $\left\{v_{n}\right\}$ such that $I+V$ and $I+V^{*}$ are hypercyclic and the corresponding weights of $L$ and $V$ are the same except, possibly, for $v_{0} \leq w_{0}, v_{4} \leq$ $w_{4}, v_{-4} \leq w_{-4}, v_{12} \leq w_{12}, v_{-20} \leq w_{-20}, v_{44} \leq w_{44}, v_{-84} \leq w_{-84}, \ldots$

We are now in a position to answer affirmatively Question 1 of [15].

COROLlaRY 4.2. The operators considered in Theorem 3.1 and Theorem 4.1 are different from the ones considered in Theorem 1 of [15].

Proof. If $T$ is as in Theorem 3.1, then $\sigma(I+T)=\{1\}$ by Proposition 2.1. Let $V$ be as in Theorem 4.1. By Remark 2.2, $\sigma(I+V)$ is one of the following: (i) $\{1\}$, (ii) a disk centered at $\{1\}$, or (iii) an annulus centered at $\{1\}$.

On the other hand, the dual hypercyclic operators considered in [15] are bilateral shifts, although not necessarily with respect to an orthonormal basis. So, in principle, those shifts are not necessarily similar to shifts with respect to an orthonormal basis. 
In any event, Proposition 2.3 asserts that these operators have spectra with circular symmetry and therefore cannot be similar to $I+T$ nor to $I+V$.

5. Final Comments. Probably it is possible to give another proof of Theorem 3.1 by showing directly that $I+T$ and $I+T^{*}$ satisfy the Hypercyclicity Criterion; Section 4 of [12] might be relevant for doing this.

The third question of [15] whether there exists a dual hypercyclic operator on a non-normable Fréċhet space remains unanswered. The papers by Ansari [2] and Bonet and Peris [7] might be relevant in this regard. The proof of Theorem 3.1 is more in the spirit of what Bernal-González did in [5]. (By using just $L_{2 k-1}$, the proof of Theorem 3.1 shows that $I+T$, where $T=\lim _{k} L_{2 k-1}$, is hypercyclic whenever $E$ is separable.)

The dual hypercyclic operator $T$ in [17] also satisfies that $T \oplus T$ is dual hypercyclic. Is $T \oplus T$ also a new kind of dual hypercyclic operator? It might be so; however, in this case the spectra argument above does not rule out the possibility that $T \oplus T$ be similar to the dual hypercyclic operators considered in [15].

Let $B \in \ell^{2}(\mathbb{N})$ be the backward shift $B\left(e_{0}\right)=0$ and $B\left(e_{n}\right)=e_{n-1}$, where $\left\{e_{n}: n \in \mathbb{N}\right\}$ is the canonical orthonormal basis. In [1], Abakumov and Gordon showed that the family $\lambda B$ with $|\lambda|>1$ has a common hypercyclic vector. It is possible that in Theorem 3.1 all the operators

$$
I+\sum_{n \in \mathbb{Z}} \mu_{n} e_{n}^{*} \otimes e_{n-1}
$$

such that all $\mu_{n}>0$ and $\mu_{n}=v_{n}$ except, possibly, for $\mu_{0} \leq v_{0}, \mu_{4} \leq v_{4}, \quad \mu_{-4} \leq$ $v_{-4}, \mu_{12} \leq w_{12}, \mu_{-20} \leq w_{-20}, \mu_{44} \leq w_{44}, \mu_{-84} \leq w_{-84} \ldots$ are hypercyclic and have a common hypercyclic vector. Moreover, they might have a common hypercyclic subspace. There have been a few papers studying common hypercyclic subspaces, see for instance the one by Bayart, [4], and the paper by Aron, Bès, León and Peris, [3]. See also Proposition 3 of [15].

Recall that a chaotic (and thus hypercyclic) operator $T$ has a dense set of periodic points [8, p263], and therefore the point spectrum $\sigma_{0}(T) \neq \emptyset$. On the other hand, it is well known that $\sigma_{0}\left(T^{*}\right)=\emptyset$ whenever $T$ is hypercyclic [14, p180]. (This fact was observed for Hilbert spaces, but it is well-known that it is true in general.) Since $\sigma_{0}(T) \subset \sigma_{0}\left(T^{* *}\right)$, there cannot be dual hypercyclic operators which are also chaotic.

The following problems are intriguing.

Problem 1: Are there any other dual hypercyclic operators other than direct sums of the ones known so far?

Problem 2: Characterize the bilateral shifts $T$ such that $I+T$ is hypercyclic.

Problem 3: Characterize the bilateral shifts $T$ such that $I+T$ is dual hypercyclic.

Acknowledgements. I would like to thank Professor Karl G. Grosse-Erdmann for useful conversations. I would also like to thank the referee for his/her helpful comments.

\section{REFERENCES}

1. E. Abakumov and J. Gordon, Common hypercyclic vectors for multiples of backward shift, J. Funct. Anal. 200 (2003), no. 2, 494-504.

2. S. I. Ansari, Existence of hypercyclic operators on topological vector spaces, J. Funct. Anal. 148 (1997), no. 2, 384-390. 
3. R. Aron, J. Bès, F. León and A. Peris, Operators with common hypercyclic subspaces, J. Operator Theory 54 (2005), no. 2, 251-260.

4. F. Bayart, Common hypercyclic subspaces, Integral Equations and Operator Theory $\mathbf{5 3}$ (2005), no. 4, 467- 476.

5. L. Bernal-González, On hypercyclic operators on Banach spaces, Proc. Amer. Math. Soc. 127 (1999), no. 4, 1003-1010.

6. J. P. Bès and A. Peris, Hereditarily hypercyclic operators, J. Funct. Anal. 167 (1999), no. 1, 94-112.

7. J. Bonet and A. Peris, Hypercyclic operators on non-normable Fréchet spaces, J. Funct. Anal. 159 (1998), no. 2, 587-595.

8. G. Godefroy and J. H. Shapiro, Operators with dense, invariant, cyclic vector manifolds J. Funct. Anal. 98 (1991), 229-269.

9. M. González, F. León-Saavedra and A. Montes-Rodríguez, Semi Fredholm theory, hypercyclic and supercyclic subspaces, Proc. London Math. Soc. 80 (2000), no 1, 169-189.

10. S. Grivaux, Hypercyclic operators, mixing operators, and the bounded steps problem, J. Operator Theory 54 (2005), no. 1, 147-168.

11. K. G. Grosse-Erdman, Universal families and hypercyclic operators, Bull. Amer. Math. Soc. (N.S.) 36 (1999), no. 3, 345-381.

12. F. León-Saavedra and A. Montes-Rodríguez, Linear structure of hypercyclic vectors, J. Funct. Anal. 148 (1997), no. 2, 524-545.

13. J. Lindenstrauss and L. Tzafriri, Classical Banach spaces. I. Sequence spaces, Ergebnisse der Mathematik und ihrer Grenzgebiete, Vol. 92 (Springer-Verlag, 1977).

14. D. A. Herrero, Limits of hypercyclic and supercyclic operators, J. Funct. Anal. 99 (1991), 179-190.

15. H. Petersson, Spaces that admit hypercyclic operators with hypercyclic adjoints, Proc. Amer. Math. Soc. 134 (2005), 1671-1676.

16. S. Rolewicz, On orbits of elements, Studia Math. 32 (1969), 17-22.

17. H. N. Salas, A hypercyclic operator whose adjoint is also hypercyclic, Proc. Amer. Math. Soc. 112 (1991), no. 3, 765-770.

18. H. N. Salas, Hypercyclic weighted shifts, Trans. Amer. Math. Soc. 347 (1995), no. 3, 993-1004.

19. A. L. Shields, Weighted shift operators and analytic function theory, Math. Survey Monographs, Vol 12 Amer. Math. Soc. Providence, RI 1974; Second Printing 1979), 49-128. 